\title{
Editorial
}

\section{Expanding the electrotherapeutic toolkit: a perspective on transcranial pulsating electromagnetic fields (T-PEMF)}

The multifocal application of transcranial lowvoltage pulsed electromagnetic fields (T-PEMF) has been shown to have an antidepressant effect in patients suffering from treatment-resistant depression (1). This proof-of-principle study had a sham-controlled, double blind study design in which sham $(n=25)$ or active $(n=25)$ T-PEMF treatment was self-administered by the patients on all weekdays for 5 weeks while pre-existing pharmacotherapy was continued. In this issue, Strass $\varnothing$ et al. (2014) (2) report the results of a randomised, double blind, dose-remission study in which patients with treatment-resistant depression self-administered a 30-min session of T-PEMF twice a day for 8 weeks. T-PEMF was administered in augmentation to their pre-existing antidepressant pharmacotherapy. In all, 34 patients received an active T-PEMF session in the morning and a sham T-PEMF in the afternoon, whereas 31 patients received two active T-PEMF sessions in the morning and afternoon. A Hamilton Depression Scale (HAM-D17) score of 7 or less defined remission. In both groups, T-PEMF treatment induced a marked remission rate in more than $65 \%$ of the patients after 8 weeks of T-PEMF treatment. Contrary to the author's expectations, there was no dose effect as remission rates were comparable for one versus two daily T-PEMF sessions. In contrast, the duration of T-PEMF treatment was found to have a strong impact on remission rates with a marked increase in remission rate by an extension of the treatment period from 5 weeks (remission rate $<33 \%$ ) to 8 weeks (remission rate $>65 \%$ ). The results imply that the remission rates following T-PEMF augmented depression therapy markedly improved as a function of treatment duration. Together, the published data suggest that T-PEMF may be a therapeutic option for therapy-resistant depression and call for larger replication studies across multiple centers. The possible neuropsychiatric application of T-PEMF has widened the therapeutic use of T-PEMF stimulation which is mainly used in orthopedics as an FDA approved technique to stimulate bone growth since 1979 $(3,4,5,6)$.

The study by Strassø et al. (2014) also points to complex dose-response relationships between the T-PEMF protocol and its anti-depressive action. This calls for future studies that unravel the neurobiological mechanisms mediating the anti-depressive effect of T-PEMF. Such studies will provide a framework according to which T-PEMF can be optimised and may identify early markers that indicate whether a patient will benefit from a prolonged T-PEMF treatment.

Electromagnetic fields and electric currents have been used as a potential cure for a wide range conditions since the time of Paracelsus (7) and the past years have evidenced a growing number of noninvasive brain stimulation (NIBS)-based neuropsychiatric therapies $(8,9)$. T-PEMF adds to the range of currently available interventional NIBS techniques such as repetitive transcranial magnetic stimulation (rTMS) (10), transcranial direct current stimulation (tDCS) and transcranial alternating current stimulation (tACS) (11), transcranial random noise stimulation (tRNS) (12), or static magnetic field stimulation (13). Future research should make an effort to compare the clinical and neurobiological effects of T-PEMF to other interventional approaches that apply constant or time-varying electric currents or electromagnetic fields through the intact skull to treat depression or other brain disorders. All these NIBS techniques are able to alter neural excitability and efficacy, but they greatly vary in three factors ultimately determining the specific effect of stimulation: the spatial distribution, the strength and the temporal variation of the electric field produced in the brain (14).

To better understand the physiological mechanisms targeted by T-PEMF compared with other NIBS techniques it is important to realise how these factors differ in T-PEMF: In contrast to all other NIBS approaches which aim at modulating a specific cortical target area, T-PEMF causes diffuse, multifocal brain stimulation of several brain regions. The T-PEMF 
device consists of a treatment helmet, which incorporates seven large stimulation coils, with complex geometry. Six coils are placed bilaterally over an anterior and posterior temporal and an upper parietal region and one coil covers the lower occipital region ((1) Supplementary material). Here the question arises whether the anti-depressive effect of T-PEMF requires this diffuse, multi-focal stimulation or whether specific coils of the seven-coil arrangement are critical to produce the therapeutic effect. Another open question is why the coils are placed over these specific brain regions. Specifically, it would be worthwhile to include prefrontal coil positions and investigate whether this would boost the therapeutic effect of T-PEMF.

T-PEMF also induces extremely low electric fields (for comparison: T-PEMF: $0.004 \mathrm{~V} / \mathrm{m}$, TMS: $90 \mathrm{~V} / \mathrm{m}$ and tDCS: $0.38 \mathrm{~V} / \mathrm{m}(6,15,16))$. This means that T-PEMF stimulation, in contrast to rTMS, stays well below the neuronal firing threshold, but may modify intrinsic brain oscillations or induce stochastic resonance. Finally, the temporal variation (stimulating frequency) of T-PEMF is higher than in most other NIBS techniques: T-PEMF uses relatively high, pulsating frequencies of around $50 \mathrm{~Hz}$. In contrast, repetitive TMS $(1-20 \mathrm{~Hz})$ and tACS $(10-20 \mathrm{~Hz})$ studies have induced electrical currents in the brain at much lower frequencies. Since the frequency of stimulation is likely to determine which neural oscillations in the brain are influenced by NIBS, a systematic study of the effect of T-PEMF frequency effects is warranted.

Even though the electric fields induced by different brain stimulations are very different, they all change cell membrane potentials to varying degrees. Thereby, they are able to influence neural excitability and synaptic efficacy via calcium-sensitive signalling mechanisms like the calcium-dependent kinase II or the cyclic adenosine monophosphate-dependent pathways. Additionally, both T-PEMF and other NIBS techniques seem to be able to elevate a range of different growth factors $(17,18,6)$.

However, the differential mechanisms governing the conversion of different electrical fields into cellular signalling events that underlie changes in neural efficacy are not yet understood.

To better understand the dosage and duration dependence of therapeutic outcome and the basic relationships between stimulation type, clinical effect and underlying biochemical processes, elementary physiological measures of NIBS after-effects like cortical excitability have to be systematically investigated. To our knowledge only a single study investigated the effect of T-PEMF on cortical excitability by measuring the amplitude of the motor evoked potential induced by single-pulse TMS of the primary motor hand area (19): They found a selective increase in intracortical facilitation, a measure related to glutamatergic activity (20), while all other parameters of cortical excitability remained unchanged. This is in contrast to the after-effects of other NIBS techniques like rTMS and tDCS. Depending on the pattern of stimulation (rTMS), or the polarity ( $\mathrm{tDCS}$ ), effects on a wide range of excitability parameters, reflecting GABAergic as well as glutamatergic neurotransmission, have been found (21).

The interesting dosage and duration effects reported in this study should be investigated in a framework of these simple after effects to be able to better understand the underlying physiology of T-PEMF. This is especially important since the final outcome of any brain stimulation protocol is influenced by a range of physiological factors such as the state of neurons before stimulation $(22,23)$ or the intrinsic properties of the network, which the stimulated neurons are a part of (24). How these factors influence the stimulation effectively as well as for mapping a dose and duration relationships these basic investigations will be needed to further guide the development of T-PEMF and other NIBS techniques as a therapeutic intervention.

A careful and systematic investigation and comparison of the basic after-effects of all NIBS techniques, including a careful mapping of responders and non-responders is also needed to solve the biggest problem hampering the development of effective, widely used brain stimulation-based therapies: All NIBS-based therapies are only producing marginally significant effects and many patients seem not to respond to the treatment. This is one of the major reasons for why, until now brain stimulation has not been able to establish itself as a major therapeutic tool. The one-size-fits all approach currently used in therapeutic NIBS might be one of the explanations for the small effect sizes on a group level. It is likely, that different stimulation dosages and parameters are optimal depending on individual physiology. Only the systematic investigation of dose-duration relationships and a strict comparison of the basic biochemical and neurophysiological effects of different NIBS techniques will allow to gain a better understanding of individually optimal NIBS dosage and stimulation patterns. It will also allow designing therapeutic studies with clear clinical a priori hypothesis concerning a wide range of stimulation parameters.

Anke N. Karabanov

Danish Research Center for Magnetic Resonance Copenhagen University Hospital Hvidovre Hvidovre, Denmark 
Hartwig R. Siebner

Danish Research Center for Magnetic Resonance Copenhagen University Hospital Hvidovre

Hvidovre, Denmark

\section{Department of Neurology, Copenhagen University Hospital Bispebjerg, Copenhagen, Denmark \\ E-mail: h.siebner@drcmr.dk}

\section{References}

1. Martiny K, Lunde M, Bech P. Transcranial low voltage pulsed electromagnetic fields in patients with treatmentresistant depression. Biol Psychiatry 2010;68:163-169.

2. Straas $\varnothing$ B, Lauritzen L, Vinberg M et al. Dose-remission of pulsating electromagnetic fields as augmentation in therapyresistant depression: a randomized, double-blind controlled study. Acta Neuropsychiatrica 2014;26:272-279.

3. Bassett CA, Mitchell SN, Gaston SR. Treatment of ununited tibial diaphyseal fractures with pulsing electromagnetic fields. J Bone Joint Surg Am 1981;63:511-523.

4. Bassett CA, Pilla AA, Pawluk RJ. A non-operative salvage of surgically-resistant pseudarthroses and non-unions by pulsing electromagnetic fields. A preliminary report. Clin Orthop Relat Res 1977:128-143.

5. MаRкоv MS. Expanding use of pulsed electromagnetic field therapies. Electromagn Biol Med 2007;26:257-274.

6. RahbeK U, Tritsaris K, Dissing S. Interactions of lowfrequency, pulsed electromagnetic fields with living tissue: biochemical responses and clinical results. Oral Biosci Med 2005:2.

7. MACKLIS RM. Magnetic healing, quackery, and the debate about the health effects of electromagnetic fields. Ann Intern Med 1993;118:376-383.

8. Lisanby SH, Kinnunen LH, Crupain MJ. Applications of TMS to therapy in psychiatry. J Clin Neurophysiol 2002;19:344-360.

9. Rossini PM, Rossi S. Transcranial magnetic stimulation: diagnostic, therapeutic, and research potential. Neurology 2007;68:484-488.

10. Hallett M. Transcranial magnetic stimulation: a primer. Neuron 2007;55:187-199.
11. Nitsche MA, Cohen LG, Wassermann EM, Priori A et al. Transcranial direct current stimulation: State of the art 2008. Brain Stimul 2008;1:206-223.

12. Terney D, Chaieb L, Moliadze V, Antal A, Paulus W. Increasing human brain excitability by transcranial highfrequency random noise stimulation. J Neurosci 2008;28: 14147-14155.

13. Oliviero A, Mordillo-Mateos L, Arias P, Panyavin I, Foffani G, Agullar J. Transcranial static magnetic field stimulation of the human motor cortex. J Physiol 2011;589:4949-4958.

14. Miranda PC. Physics of effects of transcranial brain stimulation. Handb Clin Neurol 2013;116:353-366.

15. Salvador R, Silva S, Basser PJ, Miranda PC. Determining which mechanisms lead to activation in the motor cortex: a modeling study of transcranial magnetic stimulation using realistic stimulus waveforms and sulcal geometry. Clin Neurophysiol 2011;122:748-758.

16. Miranda PC, Mekonnen A, Salvador R, Ruffini G. The electric field in the cortex during transcranial current stimulation. Neuroimage 2013;70:48-58.

17. CoоKe SF, Bliss TV. Plasticity in the human central nervous system. Brain 2006;129:1659-1673.

18. LeE JY, KIM SH, Ko AR et al. Therapeutic effects of repetitive transcranial magnetic stimulation in an animal model of Parkinson's disease. Brain Res 2013;1537:290-302.

19. Capone F, Dileone M, Profice $P$ et al. Does exposure to extremely low frequency magnetic fields produce functional changes in human brain? J Neural Transm 2009;116:257-265.

20. Chen R, Cros D, Curra A et al. The clinical diagnostic utility of transcranial magnetic stimulation: report of an IFCN committee. Clin Neurophysiol 2008;119:504-532.

21. Daskalakis ZJ, Chen R. The physiology and safety of repetitive transcranial magnetic stimulation. In: Hallett M, Chokroverty S, eds. Magnetic Stimulation in Clinical Neurophysiology. Philadelphia: Elsevier Health Sciences, 2005.

22. Karabanov A, Ziemann U, Classen J, Siebner H. Understanding homeostatic plasticity. In: Miniussi C, Paulus W, Rossini P, eds. Transcranial Brain Stimulation. Boca Raton, FL: Frontiers in Neuroscience: CRC Press, 2012; 230-244.

23. Ziemann U, Siebner HR. Modifying motor learning through gating and homeostatic metaplasticity. Brain Stimul 2008; 1:60-66.

24. Zaehle T, Rach S, Herrmann CS. Transcranial alternating current stimulation enhances individual alpha activity in human EEG. PLoS One 2010;5:e13766. 